LA-UR-13-20791

\title{
Electron spin resonance in a dilute magnon gas as a probe of magnon scattering resonances
}

\author{
Yusuke Nishida \\ Theoretical Division, Los Alamos National Laboratory, Los Alamos, New Mexico 87545, USA and \\ Department of Physics, Tokyo Institute of Technology, Ookayama, Meguro, Tokyo 152-8551, Japan
}

(Dated: February 2013)

\begin{abstract}
We study the electron spin resonance in a dilute magnon gas that is realized in a ferromagnetic spin system at low temperature. A quantum cluster expansion is developed to show that the frequency shift of the single-magnon peak changes its sign and the linewidth reaches its maximum across a scattering resonance between magnons. Such characteristic behaviors are universal and can be used to experimentally locate the two-magnon resonance when an external parameter such as pressure is varied. Future achievement of the two-magnon resonance may have an impact comparable to the Feshbach resonance in ultracold atoms and will open up a rich variety of strongly correlated physics such as the recently proposed Efimov effect in quantum magnets. We also suggest how the emergence of an Efimov state of three magnons and its binding energy may be observed with the electron spin resonance.

PACS numbers: 75.10.Jm, 75.30.Ds, 75.30.Gw, 76.30.-v
\end{abstract}

\section{INTRODUCTION}

Scattering resonances play an important role in physics. In the field of ultracold atoms, Feshbach resonances induced with magnetic field are used to control the interaction between atoms. $\frac{1}{1}$ This unparalleled tunability of the interaction has been essential to the recent remarkable advance of the field and led to experimental realization of a rich variety of physics, such as the BCS-BEC crossover in Fermi gases ${ }^{2,3}$ and the Efimov effect in Bose gases. $\frac{4}{4}$ These strongly correlated phenomena in the vicinity of the scattering resonance are universal, i.e., independent of microscopic details. Also, an atom loss peak caused by the three-atom or atom-dimer resonance has been used as a signature of the emergence of an Efimov trimer 4,5

Turning to the field of condensed matter, the scattering resonance can be induced between collective excitations in ferromagnetic spin systems (magnons), for example, by tuning the exchange coupling. $\underline{6}$ Because the ferromagnetic coupling is sensitive to the structure of ions, $\frac{7}{-}$ it is experimentally possible to vary the exchange coupling significantly with pressure ${ }^{8}$ and bring the system close to the two-magnon resonance. Future achievement of the two-magnon resonance may have an impact comparable to the Feshbach resonance in ultracold atoms and will open up a rich variety of strongly correlated physics such as the recently proposed Efimov effect in quantum magnets $\underline{6}$ But, what is an experimental signature of the two-magnon resonance?

In this paper, we study the electron spin resonance (ESR) in a dilute magnon gas as a probe of magnon scattering resonances. ESR is a powerful experimental technique to investigate the dynamics of interacting spin systems, $, 9,10$ while theoretical calculations of its spectrum are in general challenging and thus often suffer from some limitations. For example, the previous approaches developed by Kubo-Tomita ${ }^{11}$ and Mori-Kawasaki ${ }^{12}$ are based on perturbations in terms of small spin anisotropies, 13 which are actually of no use for our purpose because magnon scattering resonances take place at large spin anisotropies $\underline{\underline{6}}$ Therefore, a new approach must be developed where spin anisotropies can be treated nonperturbatively. One such approach is a quantum cluster (or virial) expansion which uses a fugacity $z \equiv e^{-\beta \Delta}$ as a small parameter to perform a systematic expansion 14 Here $\Delta$ is a single-particle excitation energy, $\beta \equiv 1 /\left(k_{\mathrm{B}} T\right)$ the inverse temperature, and the system is assumed to be so dilute that $z \ll 1$, but importantly, no assumptions are needed on the form of the interaction.

We first demonstrate this quantum cluster expansion in Sec. II by computing a single-particle spectral function in a dilute Bose gas, which serves as the universal formula for ESR at low temperature and can be used to extract the scattering length between magnons. Then in Sec. III, we apply the same method to ESR in a dilute magnon gas and show that the frequency shift of the single-magnon peak changes its sign and the linewidth reaches its maximum across a scattering resonance between magnons. Such characteristic behaviors are universal and can be used to experimentally locate the two-magnon resonance when an external parameter such as pressure is varied. Finally, Sec. IV is devoted to the conclusion of this paper and discussion where we also suggest how the emergence of an Efimov state of three magnons and its binding energy may be observed with ESR. Below we set $\hbar=k_{\mathrm{B}}=1$ and the unspecified range of integration is assumed to be from $-\infty$ to $\infty$.

\section{SINGLE-PARTICLE SPECTRAL FUNCTION IN A DILUTE BOSE GAS}

Before we work on ESR in a dilute magnon gas, it is instructive to demonstrate the quantum cluster expansion 
in a dilute Bose gas, which is described by a Hamiltonian

$$
H=\int d \boldsymbol{r} \psi_{\boldsymbol{r}}^{\dagger}\left(\varepsilon_{-i \boldsymbol{\nabla}}+\Delta\right) \psi_{\boldsymbol{r}}-\frac{g}{2} \int d \boldsymbol{r} \psi_{\boldsymbol{r}}^{\dagger} \psi_{\boldsymbol{r}}^{\dagger} \psi_{\boldsymbol{r}} \psi_{\boldsymbol{r}}
$$

Here $\varepsilon_{\boldsymbol{p}}=\boldsymbol{p}^{2} /(2 m)$ is the single-particle dispersion relation, $\mu=-\Delta<0$ is the chemical potential, and the bare coupling constant $g$ is related to the $s$-wave scattering length $a_{s}$ by $1 / g=m \Lambda /\left(2 \pi^{2}\right)-m /\left(4 \pi a_{s}\right)$ with $\Lambda$ being a momentum cutoff. Our purpose in this section is to compute the single-particle spectral function

$$
A(\omega, \boldsymbol{p})=-2 \operatorname{Im} G\left(\omega+i 0^{+}, \boldsymbol{p}\right),
$$

where $G\left(i \omega_{n}, \boldsymbol{p}\right)$ with $\omega_{n} \equiv 2 \pi n / \beta$ being the Matsubara frequency is the Fourier transform of the imaginary-time propagator

$$
G\left(i \omega_{n}, \boldsymbol{p}\right)=-\int_{0}^{\beta} d \tau \int d \boldsymbol{r} e^{i \omega_{n} \tau-i \boldsymbol{p} \cdot \boldsymbol{r}}\left\langle\psi_{\boldsymbol{r}}(\tau) \psi_{\mathbf{0}}^{\dagger}(0)\right\rangle .
$$

As we will show in the next section, $A(\omega, \mathbf{0})$ serves as the universal formula for ESR at low temperature and can be used to extract the scattering length between magnons.

The imaginary-time propagator is defined by

$$
-\left\langle\psi_{\boldsymbol{r}}(\tau) \psi_{\mathbf{0}}^{\dagger}(0)\right\rangle=-\frac{1}{Z} \operatorname{Tr}\left[e^{-\beta H} \psi_{\boldsymbol{r}}(\tau) \psi_{\mathbf{0}}^{\dagger}(0)\right]
$$

where $Z$ is the grand canonical partition function: $Z=$ $\operatorname{Tr}\left[e^{-\beta H}\right]$. The systematic expansion over the fugacity $z=e^{-\beta \Delta} \ll 1$ can be developed by writing the grand canonical trace as a sum over canonical traces with fixed particle number $N$ : $\operatorname{Tr}[\cdot]=\sum_{N=0}^{\infty} \operatorname{tr}_{N}[\cdot]$. Because $\operatorname{tr}_{N}\left[e^{-\beta H}\right] \propto z^{N}$, we obtain

$$
Z=1+V \frac{z}{\lambda^{3}}+O\left(z^{2}\right),
$$

where $V$ is the system volume and $\lambda \equiv \sqrt{2 \pi \beta / m}$ is the thermal de Broglie wavelength. Accordingly, the particle number density is found to be

$$
n=\frac{1}{V \beta} \frac{\partial \ln Z}{\partial \mu}=\frac{z}{\lambda^{3}}+O\left(z^{2}\right) .
$$

Therefore, the quantum cluster expansion is valid when the system is so dilute that the mean interparticle distance is much larger than the thermal de Broglie wavelength: $n^{-1 / 3} \gg \lambda$.

The numerator in Eq. (4) can be expanded over $z$ in the same way. By denoting the Fourier transform of each term as

$$
\begin{aligned}
G_{N}\left(i \omega_{n}, \boldsymbol{p}\right) & \equiv-\int_{0}^{\beta} d \tau \int d \boldsymbol{r} e^{i \omega_{n} \tau-i \boldsymbol{p} \cdot \boldsymbol{r}} \\
& \times \operatorname{tr}_{N}\left[e^{-\beta H} \psi_{\boldsymbol{r}}(\tau) \psi_{\mathbf{0}}^{\dagger}(0)\right] \sim O\left(z^{N}\right),
\end{aligned}
$$

the leading term is easily evaluated as

$$
G_{0}\left(i \omega_{n}, \boldsymbol{p}\right)=\frac{1-e^{-\beta \varepsilon_{\boldsymbol{p}}} z}{i \omega_{n}-\varepsilon_{\boldsymbol{p}}-\Delta} .
$$

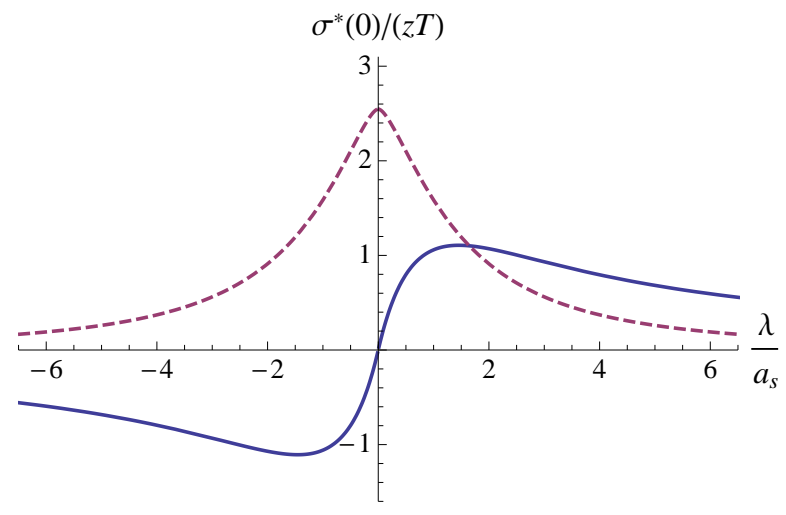

FIG. 1. Real and imaginary parts of $\sigma^{*}(\mathbf{0}) /(z T)$ in Eq. (14) as functions of $\lambda / a_{s}$, represented by solid and dashed curves, respectively.

On the other hand, after a straightforward calculation, the next-to-leading term is evaluated as

$$
\begin{aligned}
& G_{1}\left(i \omega_{n}, \boldsymbol{p}\right)=\frac{V / \lambda^{3}+e^{-\beta \varepsilon_{\boldsymbol{p}}}}{i \omega_{n}-\varepsilon_{\boldsymbol{p}}-\Delta} z \\
& +\int \frac{d \boldsymbol{q}}{(2 \pi)^{3}} \frac{F\left(i \omega_{n}, \boldsymbol{p} ; \boldsymbol{q}\right)}{\left(i \omega_{n}-\varepsilon_{\boldsymbol{p}}-\Delta\right)^{2}} e^{-\beta \varepsilon_{\boldsymbol{q}}} z+O\left(z^{2}\right),
\end{aligned}
$$

where

$$
F\left(i \omega_{n}, \boldsymbol{p} ; \boldsymbol{q}\right) \equiv \frac{8 \pi / m}{1 / a_{s}-\sqrt{-m\left(i \omega_{n}-\Delta+\varepsilon_{\boldsymbol{q}}-\varepsilon_{\boldsymbol{p}+\boldsymbol{q}} / 2\right)}}
$$

is the forward scattering amplitude between a particle with energy-momentum $\left(i \omega_{n}-\Delta, \boldsymbol{p}\right)$ and an on-shell particle with momentum $\boldsymbol{q}$. Then, by writing the Fourier transform of the imaginary-time propagator (3) in the standard form

$$
G\left(i \omega_{n}, \boldsymbol{p}\right)=\frac{1}{i \omega_{n}-\varepsilon_{\boldsymbol{p}}-\Delta-\Sigma\left(i \omega_{n}, \boldsymbol{p}\right)}
$$

and comparing it with its systematic expansion obtained in Eqs. (5), (8), and (9), we find that the self-energy $\Sigma\left(i \omega_{n}, \boldsymbol{p}\right)$ must have the following quantum cluster expansion:

$$
\Sigma\left(i \omega_{n}, \boldsymbol{p}\right)=z \int \frac{d \boldsymbol{q}}{(2 \pi)^{3}} F\left(i \omega_{n}, \boldsymbol{p} ; \boldsymbol{q}\right) e^{-\beta \varepsilon_{\boldsymbol{q}}}+O\left(z^{2}\right) .
$$

Therefore, the self-energy at $O(z)$ is determined only by the two-particle physics, i.e., binary collisions with thermally excited particles.

The resulting single-particle spectral function (2) at $O\left(z^{0}\right)$ is simply a delta function located at $\omega=\varepsilon_{\boldsymbol{p}}+\Delta$ corresponding to the single-particle energy. By including the self-energy correction $\Sigma\left(i \omega_{n}, \boldsymbol{p}\right) \sim O(z) \ll 1$, it becomes a sharp peak whose line shape within the accuracy up to $O(z)$ is described by the Lorentzian

$$
A_{\text {peak }}(\omega, \boldsymbol{p}) \approx \frac{-2 \operatorname{Im} \sigma(\boldsymbol{p})}{\left[\omega-\varepsilon_{\boldsymbol{p}}-\Delta-\operatorname{Re} \sigma(\boldsymbol{p})\right]^{2}+[\operatorname{Im} \sigma(\boldsymbol{p})]^{2}},
$$




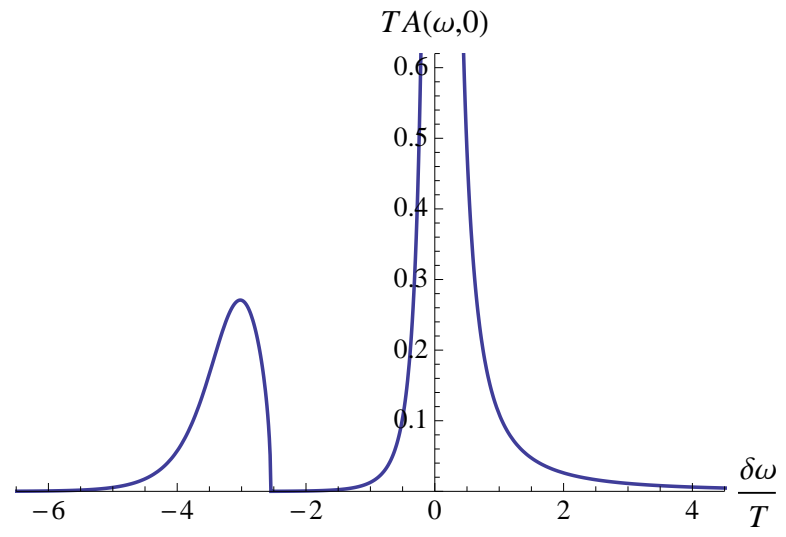

FIG. 2. Single-particle spectral function $A(\omega, \mathbf{0})$ for $z=0.1$ and $\lambda / a_{s}=4$ as a function of $\delta \omega \equiv \omega-\Delta$. The singleparticle peak goes up to $T A(\omega, \mathbf{0}) \simeq 51$ and there is a threshold singularity at the two-particle binding energy $E_{2} / T=$ $-\left(\lambda / a_{s}\right)^{2} /(2 \pi)$.

where we introduced the on-shell self-energy

$$
\begin{aligned}
\sigma(\boldsymbol{p}) & \equiv \Sigma\left(\varepsilon_{\boldsymbol{p}}+\Delta+i 0^{+}, \boldsymbol{p}\right) \\
& =z \int \frac{d \boldsymbol{q}}{(2 \pi)^{3}} \frac{8 \pi / m}{1 / a_{s}+i|\boldsymbol{p}-\boldsymbol{q}| / 2} e^{-\beta \varepsilon_{\boldsymbol{q}}} .
\end{aligned}
$$

Therefore, the energy shift and the decay width of a particle with momentum $\boldsymbol{p}$ in a dilute Bose gas are given by the real and imaginary parts of $\sigma^{*}(\boldsymbol{p})$, respectively. The corresponding normalized quantity $\sigma^{*}(\boldsymbol{p}) /(z T) \sim O(1)$ at $\boldsymbol{p}=\mathbf{0}$ is plotted in Fig. 11 as a function of $\lambda / a_{s}$. We find that the energy shift changes its sign and the decay width reaches its maximum across the scattering resonance at $a_{s} \rightarrow \infty$. Such characteristic behaviors are universal and shared by ESR at low temperature.

Besides the single-particle peak at $\omega=\varepsilon_{\boldsymbol{p}}+\Delta+$ $\operatorname{Re} \sigma(\boldsymbol{p})$, there exists an additional structure in $A(\omega, \boldsymbol{p})$ at $\omega<\varepsilon_{p}+\Delta+E_{2}$ when two particles form a bound state with binding energy $E_{2}=-1 /\left(m a_{s}^{2}\right)<0$. This structure is due to the pole of the two-particle scattering amplitude (10) and shows a square-root threshold singularity at $\omega=\varepsilon_{\boldsymbol{p}}+\Delta+E_{2}$ in the single-particle spectral function as $A(\omega, \boldsymbol{p}) \sim z \sqrt{\varepsilon_{\boldsymbol{p}}+\Delta+E_{2}-\omega}$. This feature at $\boldsymbol{p}=\mathbf{0}$ is demonstrated in Fig. 2 by choosing $z=0.1$ and $\lambda / a_{s}=4$ as an example. We also note that the single-particle spectrum function of bosons is universal up to $O\left(z^{2}\right)$, i.e., independent of an ultraviolet cutoff. The $O\left(z^{2}\right)$ term involves the three-particle physics and thus depends on an additional parameter, so-called Efimov parameter $\kappa_{*}, \frac{15}{5}$ See the discussion in Sec. IV on how the emergence of an Efimov state and its binding energy may appear in the expected behavior of $A(\omega, \mathbf{0})$ at $O\left(z^{2}\right)$.

\section{ELECTRON SPIN RESONANCE IN A DILUTE MAGNON GAS}

\section{A. Quantum cluster expansion}

We now apply the same method to ESR in a dilute magnon gas. For definiteness, we consider a spin$S$ Heisenberg model with exchange $\left(J_{z} \neq J>0\right)$ and single-ion $(D \neq 0)$ anisotropies, which is described by

$$
\begin{aligned}
H= & -\frac{1}{2} \sum_{\boldsymbol{r}} \sum_{\hat{e}}\left(J S_{\boldsymbol{r}}^{+} S_{\boldsymbol{r}+\hat{\boldsymbol{e}}}^{-}+J_{z} S_{\boldsymbol{r}}^{z} S_{\boldsymbol{r}+\hat{e}}^{z}\right) \\
& -D \sum_{\boldsymbol{r}}\left(S_{\boldsymbol{r}}^{z}\right)^{2}-B \sum_{\boldsymbol{r}} S_{\boldsymbol{r}}^{z} .
\end{aligned}
$$

Here $\sum_{\hat{\boldsymbol{e}}}=\sum_{\hat{\boldsymbol{e}}= \pm \hat{\boldsymbol{x}}, \pm \hat{\boldsymbol{y}}, \pm \hat{\boldsymbol{z}}}$ is a sum over six unit vectors of a simple cubic lattice and spin operators $S_{r}^{ \pm} \equiv$ $S_{r}^{x} \pm i S_{r}^{y}$ and $S_{r}^{z}$ obey the usual commutation relations: $\left[S_{\boldsymbol{r}}^{+}, S_{\boldsymbol{r}^{\prime}}^{-}\right]=2 S_{\boldsymbol{r}}^{z} \delta_{\boldsymbol{r}, \boldsymbol{r}^{\prime}}$ and $\left[S_{\boldsymbol{r}}^{z}, S_{\boldsymbol{r}^{\prime}}^{ \pm}\right]= \pm S_{\boldsymbol{r}}^{ \pm} \delta_{\boldsymbol{r}, \boldsymbol{r}^{\prime}}$. ESR experiments measure an absorption intensity of electromagnetic radiation polarized perpendicular to the magnetic field axis. Within the linear response theory for a circular polarization, the absorption intensity normalized by the system volume and the intensity of the incident radiation is given by $\underline{13,16}$

$$
I(\omega)=\frac{\omega}{2} \operatorname{Im} \chi\left(\omega+i 0^{+}, \mathbf{0}\right),
$$

where $\chi\left(i \omega_{n}, \boldsymbol{p}\right)$ is the Fourier transform of the imaginary-time susceptibility

$$
\chi\left(i \omega_{n}, \boldsymbol{p}\right)=\int_{0}^{\beta} d \tau \sum_{\boldsymbol{r}} e^{i \omega_{n} \tau-i \boldsymbol{p} \cdot \boldsymbol{r}}\left\langle S_{\boldsymbol{r}}^{-}(\tau) S_{\mathbf{0}}^{+}(0)\right\rangle .
$$

While the ESR experiments can measure the spectrum only at zero momentum, we develop the formulation for general $\boldsymbol{p}$ and set $\boldsymbol{p}=\mathbf{0}$ at the end.

The ground state for a sufficiently large magnetic field $B<0$ is a fully polarized state with all spins pointing downwards: $S_{\boldsymbol{r}}^{z}|0\rangle=-S|0\rangle$ and $S_{\boldsymbol{r}}^{-}|0\rangle=0$. Accordingly, we redefine the Hamiltonian to absorb the ground state energy $E_{0}=-\sum_{\boldsymbol{r}}\left(3 J_{z} S^{2}+D S^{2}-B S\right)$ so that $H|0\rangle=$ 0 . Because of the U(1) symmetry under rotation $S_{r}^{ \pm} \rightarrow$ $e^{ \pm i \theta} S_{r}^{ \pm}$, the magnetization relative to the ground state $\delta M \equiv\left\langle S_{\boldsymbol{r}}^{z}\right\rangle+S$ is a conserved quantity which corresponds to a particle number density of magnons. Then at low temperature, magnons are thermally excited and thus the system becomes a dilute magnon gas. A single magnon has the dispersion relation $\varepsilon_{\boldsymbol{p}}=S J \sum_{\hat{\boldsymbol{e}}}[1-\cos (\boldsymbol{p} \cdot \hat{\boldsymbol{e}})]$ with the excitation energy $\Delta=-6 S J+6 S J_{z}+2 S D-$ $D-B$. As long as the fugacity is small, $z=e^{-\beta \Delta} \ll 1$, the quantum cluster expansion can be developed for the dilute magnon gas similarly to the previous dilute Bose gas. The imaginary-time susceptibility is defined by

$$
\left\langle S_{\boldsymbol{r}}^{-}(\tau) S_{\mathbf{0}}^{+}(0)\right\rangle=\frac{1}{Z} \operatorname{Tr}\left[e^{-\beta H} S_{\boldsymbol{r}}^{-}(\tau) S_{\mathbf{0}}^{+}(0)\right],
$$


where $Z$ is the grand canonical partition function: $Z=$ $\operatorname{Tr}\left[e^{-\beta H}\right]$. By writing the grand canonical trace as a sum over canonical traces with fixed magnon number $N$, we obtain

$$
Z=\sum_{N=0}^{\infty} \operatorname{tr}_{N}\left[e^{-\beta H}\right]=1+V \frac{z}{\rho^{3}}+O\left(z^{2}\right),
$$

where we used $\operatorname{tr}_{N}\left[e^{-\beta H}\right] \propto z^{N}$ and introduced the analog of the thermal de Broglie wavelength by $\rho \equiv$ $a e^{2 \beta S J} / I_{0}(2 \beta S J)$ with $a$ being the lattice constant. Accordingly, the relative magnetization is found to be

$$
\delta M=\frac{1}{V \beta} \frac{\partial \ln Z}{\partial B}=\frac{z}{\rho^{3}}+O\left(z^{2}\right)
$$

The numerator in Eq. (18) can be expanded over $z$ in the same way. By denoting the Fourier transform of each term as

$$
\begin{aligned}
\chi_{N}\left(i \omega_{n}, \boldsymbol{p}\right) & \equiv \int_{0}^{\beta} d \tau \sum_{\boldsymbol{r}} e^{i \omega_{n} \tau-i \boldsymbol{p} \cdot \boldsymbol{r}} \\
& \times \operatorname{tr}_{N}\left[e^{-\beta H} S_{\boldsymbol{r}}^{-}(\tau) S_{\mathbf{0}}^{+}(0)\right] \sim O\left(z^{N}\right),
\end{aligned}
$$

the leading term is easily evaluated as

$$
\chi_{0}\left(i \omega_{n}, \boldsymbol{p}\right)=-2 S \frac{1-e^{-\beta \varepsilon_{\boldsymbol{p}} z}}{i \omega_{n}-\varepsilon_{\boldsymbol{p}}-\Delta} .
$$

On the other hand, after a straightforward calculation, the next-to-leading term is evaluated as

$$
\begin{aligned}
& \chi_{1}\left(i \omega_{n}, \boldsymbol{p}\right)=-2 S \frac{V / \rho^{3}+e^{-\beta \varepsilon_{\boldsymbol{p}}}}{i \omega_{n}-\varepsilon_{\boldsymbol{p}}-\Delta} z \\
& -2 S \int_{-\pi / a}^{\pi / a} \frac{d \boldsymbol{q}}{(2 \pi / a)^{3}} \frac{\Gamma\left(i \omega_{n}, \boldsymbol{p} ; \boldsymbol{q}\right)}{\left(i \omega_{n}-\varepsilon_{\boldsymbol{p}}-\Delta\right)^{2}} e^{-\beta \varepsilon_{\boldsymbol{q}}} z+O\left(z^{2}\right),
\end{aligned}
$$

where

$$
\begin{aligned}
\Gamma\left(i \omega_{n}, \boldsymbol{p} ; \boldsymbol{q}\right) \equiv & \sum_{\hat{\boldsymbol{e}}}\left[J \cos \left(\frac{\boldsymbol{p}+\boldsymbol{q}}{2} \cdot \hat{\boldsymbol{e}}\right)-J_{z} \cos \left(\frac{\boldsymbol{p}-\boldsymbol{q}}{2} \cdot \hat{\boldsymbol{e}}\right)\right] \gamma(\hat{\boldsymbol{e}}) \\
& -2 D \gamma(\mathbf{0})
\end{aligned}
$$

is the forward scattering amplitude between a magnon with energy-momentum $\left(i \omega_{n}-\Delta, \boldsymbol{p}\right)$ and an on-shell magnon with momentum $\boldsymbol{q}$. Here the unknown function $\gamma(\boldsymbol{r})=\gamma(-\boldsymbol{r})$ implicitly depends on $\left(i \omega_{n}, \boldsymbol{p} ; \boldsymbol{q}\right)$ and satisfies the Lippmann-Schwinger equation

$$
\begin{aligned}
& \gamma(\boldsymbol{r})=2 \cos \left(\frac{\boldsymbol{p}-\boldsymbol{q}}{2} \cdot \boldsymbol{r}\right)+\int_{-\pi / a}^{\pi / a} \frac{d \boldsymbol{k}}{(2 \pi / a)^{3}} \cos (\boldsymbol{k} \cdot \boldsymbol{r}) \\
& \times \frac{\sum_{\hat{\boldsymbol{e}}}\left[J \cos \left(\frac{\boldsymbol{p}+\boldsymbol{q}}{2} \cdot \hat{\boldsymbol{e}}\right)-J_{z} \cos (\boldsymbol{k} \cdot \hat{\boldsymbol{e}})\right] \gamma(\hat{\boldsymbol{e}})-2 D \gamma(\mathbf{0})}{i \omega_{n}-\Delta+\varepsilon_{\boldsymbol{q}}-\varepsilon_{(\boldsymbol{p}+\boldsymbol{q}) / 2+\boldsymbol{k}}-\varepsilon_{(\boldsymbol{p}+\boldsymbol{q}) / 2-\boldsymbol{k}}} .
\end{aligned}
$$

By setting $\boldsymbol{r}=\hat{\boldsymbol{x}}, \hat{\boldsymbol{y}}, \hat{\boldsymbol{z}}$ and $\boldsymbol{r}=\mathbf{0}$, we obtain four coupled equations to determine $\gamma(\hat{\boldsymbol{e}})$ and $\gamma(\mathbf{0})$ appearing in Eq. (24).
Then, by writing the Fourier transform of the imaginary-time susceptibility (17) in the standard form

$$
\chi\left(i \omega_{n}, \boldsymbol{p}\right)=\frac{-2 S}{i \omega_{n}-\varepsilon_{\boldsymbol{p}}-\Delta-\Xi\left(i \omega_{n}, \boldsymbol{p}\right)}
$$

and comparing it with its systematic expansion obtained in Eqs. (19), (22), and (23), we find that the self-energy $\Xi\left(i \omega_{n}, \boldsymbol{p}\right)$ must have the following quantum cluster expansion:

$$
\Xi\left(i \omega_{n}, \boldsymbol{p}\right)=z \int_{-\pi / a}^{\pi / a} \frac{d \boldsymbol{q}}{(2 \pi / a)^{3}} \Gamma\left(i \omega_{n}, \boldsymbol{p} ; \boldsymbol{q}\right) e^{-\beta \varepsilon_{\boldsymbol{q}}}+O\left(z^{2}\right) .
$$

Therefore, the self-energy at $O(z)$ is determined only by the two-magnon physics, i.e., binary collisions with thermally excited magnons. The resulting ESR spectrum (16) at $O\left(z^{0}\right)$ is simply a delta function located at $\omega=\Delta$ corresponding to the single-magnon energy at $\boldsymbol{p}=\mathbf{0}$. By including the self-energy correction $\Xi\left(i \omega_{n}, \mathbf{0}\right) \sim O(z) \ll$ 1 , it becomes a sharp peak whose line shape within the accuracy up to $O(z)$ is described by the Lorentzian

$$
I_{\text {peak }}(\omega) \approx \frac{\omega}{2} \frac{-2 S \operatorname{Im} \xi(\mathbf{0})}{[\omega-\Delta-\operatorname{Re} \xi(\mathbf{0})]^{2}+[\operatorname{Im} \xi(\mathbf{0})]^{2}},
$$

where we introduced the on-shell self-energy: $\xi(\boldsymbol{p}) \equiv$ $\Xi\left(\varepsilon_{\boldsymbol{p}}+\Delta+i 0^{+}, \boldsymbol{p}\right)$. Therefore, the frequency shift and the linewidth of the single-magnon peak are given by the real and imaginary parts of $\xi^{*}(\mathbf{0})$, respectively. ${ }^{17}$ Also, when two magnons form a bound state with binding energy $E_{2}<0$, the ESR spectrum shows an additional structure at $\omega<\Delta+E_{2}$ similarly to Fig. 2, while we will not investigate it further.

\section{B. Solution and results}

Our remaining task is to solve the LippmannSchwinger equation (25) with $i \omega_{n}=\Delta+i 0^{+}$and $\boldsymbol{p}=\mathbf{0}$ :

$$
\begin{aligned}
& \gamma(\boldsymbol{r})=2 \cos \left(\frac{\boldsymbol{q}}{2} \cdot \boldsymbol{r}\right)+\int_{-\pi / a}^{\pi / a} \frac{d \boldsymbol{k}}{(2 \pi / a)^{3}} \cos (\boldsymbol{k} \cdot \boldsymbol{r}) \\
& \times \frac{\sum_{\hat{\boldsymbol{e}}}\left[J \cos \left(\frac{\boldsymbol{q}}{2} \cdot \hat{\boldsymbol{e}}\right)-J_{z} \cos (\boldsymbol{k} \cdot \hat{\boldsymbol{e}})\right] \gamma(\hat{\boldsymbol{e}})-2 D \gamma(\mathbf{0})}{\varepsilon_{\boldsymbol{q}}-\varepsilon_{\boldsymbol{q} / 2+\boldsymbol{k}}-\varepsilon_{\boldsymbol{q} / 2-\boldsymbol{k}}+i 0^{+}}
\end{aligned}
$$

to determine $\gamma(\hat{\boldsymbol{e}})$ and $\gamma(\mathbf{0})$ in the two-magnon scattering amplitude (24). In the low-temperature limit $T \rightarrow 0$, the integration over $\boldsymbol{q}$ in the self-energy (27) is dominated by the region $\boldsymbol{q} \simeq \mathbf{0}$ because of the Boltzmann factor. In this case, by expanding the right-hand side of Eq. (29) up to $O(\boldsymbol{q})$, we find $\gamma(\hat{\boldsymbol{x}})=\gamma(\hat{\boldsymbol{y}})=\gamma(\hat{\boldsymbol{z}})$ and the integration over $\boldsymbol{k}$ can be performed analytically. Then, by substituting the obtained analytical solutions for $\gamma(\hat{\boldsymbol{e}})$ and $\gamma(\mathbf{0})$ into Eq. (24), we find that the two-magnon scattering amplitude takes the same form as that of bosons 

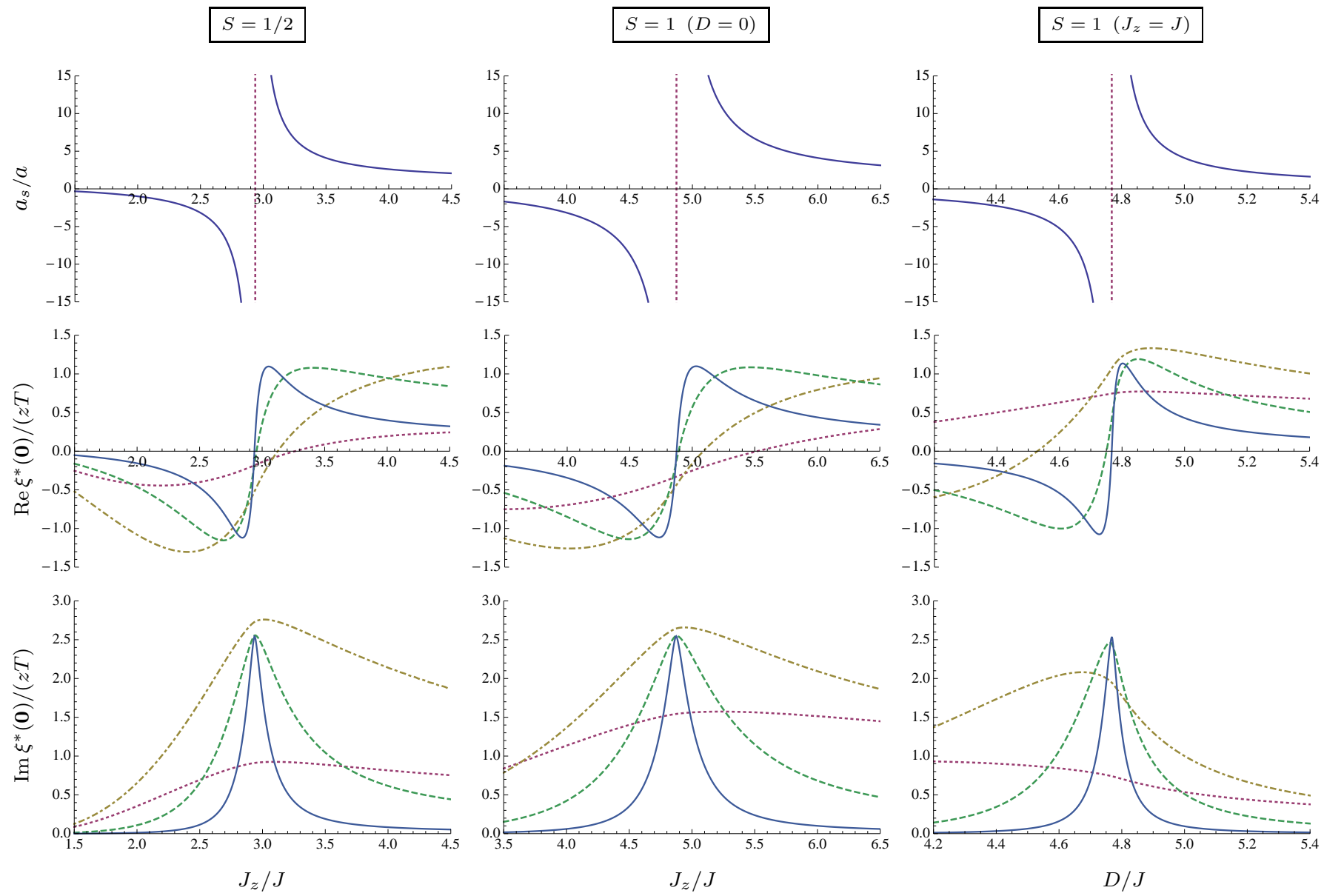

FIG. 3. Top panels show scattering lengths in Eq. (31) for $S=1 / 2$ (left), $S=1$ with $D=0$ (middle) as functions of $J_{z} / J$, and $S=1$ with $J_{z}=J$ (right) as a function of $D / J$. The vertical lines indicate the locations of two-magnon resonances where $a_{s} \rightarrow \infty$. Middle and bottom panels show real and imaginary parts of $\xi^{*}(\mathbf{0}) /(z T)$ in Eq. (28) at $T / J=0.01,0.1,1$, and 10 , represented by solid, dashed, dash-dotted, and dotted curves, respectively.

in Eq. (10):

$$
\begin{aligned}
\lim _{\boldsymbol{q} \rightarrow \mathbf{0}} \Gamma\left(\Delta+i 0^{+}, \mathbf{0} ; \boldsymbol{q}\right) & =\frac{1}{a^{3}} \frac{8 \pi / m}{1 / a_{s}+i|\boldsymbol{q}| / 2} \\
& =\frac{1}{a^{3}} F\left(\Delta+i 0^{+}, \mathbf{0} ; \boldsymbol{q}\right),
\end{aligned}
$$

where $1 / m=2 S J a^{2}$ is the inverse effective mass of magnons and

$$
\frac{a_{s}}{a}=\frac{\frac{3}{2 \pi}\left[1-\frac{D}{3 J}-\frac{J_{z}}{J}\left(1-\frac{D}{6 S J}\right)\right]}{2 S-1+\frac{J_{z}}{J}\left(1-\frac{D}{6 S J}\right)+3 W\left[1-\frac{D}{3 J}-\frac{J_{z}}{J}\left(1-\frac{D}{6 S J}\right)\right]}
$$

is the scattering length between magnons with

$$
\begin{aligned}
W & \equiv \int_{-\pi / a}^{\pi / a} \frac{d \boldsymbol{k}}{(2 \pi / a)^{3}} \frac{2}{\sum_{\hat{\boldsymbol{e}}}[1-\cos (\boldsymbol{k} \cdot \hat{\boldsymbol{e}})]} \\
& =\frac{\sqrt{6}}{96 \pi^{3}} \Gamma\left(\frac{1}{24}\right) \Gamma\left(\frac{5}{24}\right) \Gamma\left(\frac{7}{24}\right) \Gamma\left(\frac{11}{24}\right)
\end{aligned}
$$

being the Watson's triple integral for a simple cubic lattice $\underline{18}$ We note that the same scattering length was obtained in Ref. 6 with a different approach.

Accordingly, the low-temperature limit of the on-shell self-energy at $\boldsymbol{p}=\mathbf{0}$ appearing in Eq. (28) reduces to that in Eq. (14),

$$
\lim _{T \rightarrow 0} \xi(\mathbf{0})=\sigma(\mathbf{0}),
$$

and thus the line shape of the single-magnon peak is described by the universal formula

$$
\lim _{T \rightarrow 0} I_{\text {peak }}(\omega)=\frac{\omega}{2} S A_{\text {peak }}(\omega, \mathbf{0}),
$$

where $A_{\text {peak }}(\omega, \boldsymbol{p})$ is the single-particle spectral function of bosons obtained in the previous section as Eq. (13). This result is actually expected because the magnon gas at low temperature is so dilute that the system becomes independent of microscopic details and thus described by only a few low-energy parameters such as $m$ and $a_{s}$ [additionally $\kappa_{*}$ at $O\left(z^{2}\right)$ ]. Therefore, it is possible to extract the scattering length between magnons by fitting 
the universal formula (33) to the experimentally measured temperature dependence of the line shape of the single-magnon peak.

Away from the low-temperature limit, the line shape (28) is model dependent and has to be evaluated numerically by solving Eq. (29) for general $\boldsymbol{q}$. The frequency shift and the linewidth of the single-magnon peak are given by the real and imaginary parts of $\xi^{*}(\mathbf{0})$, respectively, and the corresponding normalized quantity $\xi^{*}(\mathbf{0}) /(z T) \sim O(1)$ is plotted in Fig. 3. For demonstration, we choose three distinct cases where $S=1 / 2$, $S=1$ with $D=0$ as functions of $J_{z} / J$, and $S=1$ with $J_{z}=J$ as a function of $D / J$ at four different temperatures, $T / J=0.01,0.1,1,10$. Figure 3 also displays the corresponding scattering length (31) which indicates that the two-magnon resonances $a_{s} \rightarrow \infty$ are located at $J_{z} / J=2.94, J_{z} / J=4.87$, and $D / J=4.77$, respectively, where magnons interact strongly. We find that the line shape of the single-magnon peak is well described by the universal formula (33) at low temperature $T<J$ and thus the frequency shift changes its sign and the linewidth reaches its maximum across the two-magnon resonance. Such characteristic behaviors become sharper with decreasing temperature and can be seen moderately even at intermediate temperature $T \simeq J$, while they disappear at higher temperature $T>J$.

\section{CONCLUSION AND DISCUSSION}

In this paper, we studied ESR in a dilute magnon gas that is realized in a ferromagnetic spin system at low temperature. We developed the quantum cluster expansion up to $O(z)$ which is determined by the twomagnon physics and showed that the frequency shift of the single-magnon peak changes its sign and the linewidth reaches its maximum across a scattering resonance between magnons. Such characteristic behaviors are universal and can be used to experimentally locate the two-magnon resonance when an external parameter such as pressure is varied. Future achievement of the twomagnon resonance may have an impact comparable to the Feshbach resonance in ultracold atoms and will open up a rich variety of strongly correlated physics such as the recently proposed Efimov effect in quantum magnets $\underline{\underline{6}}$

It is straightforward in principle to continue this systematic expansion to include higher-order corrections. In particular, the $O\left(z^{2}\right)$ term involves the three-magnon physics and thus it is possible to probe the Efimov ef- fect with ESR. When the system comes across the critical coupling where an Efimov state of three magnons emerges from the scattering threshold, the linewidth of the single-magnon peak as a function of the external parameter is expected to show an additional peak structure caused by the three-magnon resonance on either side of the two-magnon resonance. This feature is in analogy with ultracold atom experiments where an atom loss peak caused by the three-atom or atom-dimer resonance has been used as a signature of the emergence of an Efimov trimer.$\frac{4.5}{5}$ Similarly, a pair of universal fourmagnon states associated with every Efimov state 19,20 may be observed with ESR through an additional peak structure in the linewidth at $O\left(z^{3}\right)$ caused by the fourmagnon resonance $\stackrel{21.22}{ }$ Besides such characteristic behaviors in the single-magnon peak, we expect an additional structure in the ESR spectrum at $\omega<\Delta+E_{N}$ when $N$ magnons form a bound state with binding energy $E_{N}<0$. This structure shows a threshold singularity at $\omega=\Delta+E_{N}$ as $I(\omega) \sim z^{N-1}\left(\Delta+E_{N}-\omega\right)^{(3 N-5) / 2}$, which may be used to measure binding energies of magnon Efimov states. Therefore, ESR is a powerful experimental technique to investigate the interaction among magnons and their spectrum.

So far we considered ferromagnetic spin systems where the single-magnon dispersion relation has a minimum at zero momentum. On the other hand, it is also possible to induce the two-magnon resonance and thus the magnon Efimov effect in spin systems with antiferromagnetic or frustrated exchange couplings where the singlemagnon dispersion relation has a minimum at nonzero momentum $\underline{6,23,24}$ In these cases, however, the sharp signature of magnon scattering resonances discussed in this paper will not appear in ESR because it measures the spectrum only at zero momentum. Therefore, different experimental techniques such as inelastic neutron scattering that can scan momentum space should be used to probe magnon scattering resonances. The quantum cluster expansion developed in this paper will be useful to compute any other physical observables in a dilute magnon gas.

\section{ACKNOWLEDGMENTS}

The author thanks C. D. Batista and Y. Kato and acknowledges many valuable discussions during his visit to RIKEN and YITP in the fall of 2012. This work was supported by a LANL Oppenheimer Fellowship and JSPS KAKENHI Grant Number 25887020.
1 C. Chin, R. Grimm, P. Julienne, and E. Tiesinga, Rev. Mod. Phys. 82, 1225 (2010).

2 C. A. Regal, M. Greiner, and D. S. Jin, Phys. Rev. Lett. 92, 040403 (2004).
${ }^{3}$ M. W. Zwierlein, C. A. Stan, C. H. Schunck, S. M. F. Raupach, A. J. Kerman, and W. Ketterle, Phys. Rev. Lett. 92, 120403 (2004). 
4 T. Kraemer, M. Mark, P. Waldburger, J. G. Danzl, C. Chin, B. Engeser, A. D. Lange, K. Pilch, A. Jaakkola, H.-C. Nägerl, and R. Grimm, Nature (London) 440, 315 (2006).

5 S. Knoop, F. Ferlaino, M. Mark, M. Berninger, H. Schöbel, H.-C. Nägerl, and R. Grimm, Nat. Phys. 5, 227 (2009).

6 Y. Nishida, Y. Kato, and C. D. Batista, Nat. Phys. 9, 93 (2013).

7 J. B. Goodenough, Magnetism and the Chemical Bond (Wiley, New York, 1963).

8 T. Kawamoto, M. Tokumoto, H. Sakamoto, and K. Mizoguchi, J. Phys. Soc. Jpn. 70, 1892 (2001).

${ }^{9}$ K. Katsumata, J. Phys.: Condens. Matter 12, R589 (2000).

10 Y. Ajiro, J. Phys. Soc. Jpn. 72, 12 (2003).

${ }^{11}$ R. Kubo and K. Tomita, J. Phys. Soc. Jpn. 9, 888 (1954).

12 H. Mori and K. Kawasaki, Prog. Theor. Phys. 27, 529 (1962); Prog. Theor. Phys. 28, 971 (1962).

13 M. Oshikawa and I. Affleck, Phys. Rev. B 65, 134410 (2002).

14 L. D. Landau and E. M. Lifshitz, Statistical Physics (Butterworth-Heinemann, Oxford, 1980).

15 E. Braaten and H.-W. Hammer, Phys. Rep. 428, 259 (2006).
16 M. Brockmann, F. Göhmann, M. Karbach, A. Klümper, and A. Weiße, Phys. Rev. B 85, 134438 (2012).

17 In the absence of the spin anisotropies $J_{z}=J$ and $D=$ 0 , the two-magnon scattering amplitude (24) at $\boldsymbol{q}=\mathbf{0}$ vanishes so that the ESR spectrum shows no frequency shift and linewidth, which is consistent with the general argument given in Ref. 13. Also, by expanding Eqs. (24) and (25) in terms of the small spin anisotropies $\epsilon \sim J_{z} / J-$ $1, D / J \ll 1$, it is easy to find that the frequency shift and the linewidth are $\operatorname{Re} \xi^{*}(\mathbf{0}) \sim O(\epsilon)$ and $\operatorname{Im} \xi^{*}(\mathbf{0}) \sim O\left(\epsilon^{2}\right)$, respectively, which are again consistent with the previous results (Ref. 11-13).

18 G. N. Watson, Q. J. Math. 10, 266 (1939).

19 H.-W. Hammer and L. Platter, Eur. Phys. J. A 32, 113 (2007).

20 J. von Stecher, J. P. D'Incao, and C. H. Greene, Nat. Phys. 5, 417 (2009).

21 F. Ferlaino, S. Knoop, M. Berninger, W. Harm, J. P. D'Incao, H.-C. Nägerl, and R. Grimm, Phys. Rev. Lett. 102, 140401 (2009).

${ }^{22}$ F. Ferlaino and R. Grimm, Physics 3, 9 (2010).

${ }^{23}$ H. T. Ueda and K. Totsuka, Phys. Rev. B 80, 014417 (2009).

24 H. T. Ueda and T. Momoi, Phys. Rev. B 87, 144417 (2013). 